\title{
Utilisation des études étrangères : transposition des résultats, prédiction des effets thérapeutiques en population française, modélisation de l'Intérêt de Santé Publique
} \author{
de Giens XXI^ \\ 1 Centre Hospitalo Universitaire de Besançon, Hôpital Saint Jacques, Besançon, France \\ 2 Lilly France, Suresnes, France \\ 3 Merck Sharpe and Dohme, Paris, France
}

Jacques Massol ${ }^{1}$, Myriam Zylberman ${ }^{2}$, Jean-Marie Goehrs ${ }^{3}$ et les participants à la table ronde $n^{\circ} 1$

\begin{abstract}
Mots clés :
médicaments ;

transposabilité ;

généralisation ;

impact de santé

publique;

facteurs ethniques

Résumé - De plus en plus souvent, les autorités de santé et les agences d'évaluation françaises sont conduites à délivrer des AMM (Autorisation de Mise sur le Marché), à rendre des avis sur l'admission au remboursement de médicaments ou à élaborer des recommandations pour la pratique clinique à partir de résultats d'études étrangères. Les résultats de ces études sont plus ou moins difficiles à transposer à la pratique française. Ces difficultés génèrent une incertitude de degré variable sur l'effet à attendre d'un médicament. Une perte d'effet plus ou moins importante est quelquefois même prévisible. Quelques unes des difficultés de transposition sont discutées dans cet article et des propositions d'actions sont formulées pour permettre, à terme, de prédire de la façon la plus précise possible les effets à attendre d'un médicament en population française et de pouvoir vérifier cette prédiction à distance de son admission au remboursement.
\end{abstract}

\section{Introduction}

Les médicaments font l'objet de plans de développement internationaux, conçus pour obtenir des autorisations de mise sur le marché et un remboursement dans de nombreux pays. Placés sous le contrôle de la FDA (Food and Drug Administration) aux USA, ces plans de développement sont, en France, sous la responsabilité des entreprises pharmaceutiques. Or, force est de constater qu'à ce jour, les études cliniques issues de ces plans de développement, même lorsqu'elles sont multinationales, ne comprennent, bien souvent pas de patient français. Ce manque de représentation n'est d'ailleurs pas sans rapport avec la diminution des essais cliniques de promotion industrielle réalisés sur notre territoire qui alimente le débat de la perte d'attractivité de la France dans le domaine de la recherche ${ }^{[1]}$ et l'apparition de pays émergents en matière de recherche clinique ne laisse pas augurer d'un renversement spontané de situation.

\footnotetext{
* Pour la liste des participants, voir en fin d'article.
}

Les autorités de santé et les agences d'évaluation françaises doivent donc fonder de plus en plus souvent les décisions collectives d'enregistrement et de remboursement voire les recommandations pour la pratique clinique sur des données issues d'études étrangères. La publication de telles études expose par ailleurs, à un échelon plus individuel, au risque que le praticien, de plus en plus incité à se référer à la littérature médicale internationale, s'en approprie les conclusions sans s'interroger sur leur applicabilité à sa propre pratique.

En quoi l'origine étrangère des études pose-t-elle des difficultés de transposition? Le caractère étranger des études ne constitue, à vrai dire, qu'un élément de la question méthodologique, largement débattue par ailleurs, de la transposabilité/applicabilité des résultats des études cliniques à la pratique clinique ${ }^{[2]}$ ou des études médico-économiques. ${ }^{[3]}$

Etudiés sur des groupes de patients sélectionnés, très (trop ?) homogènes, pris en charge dans des conditions généralement éloignées de la pratique clinique, les médicaments sont cependant le plus souvent destinés à des populations diverses, larges et 


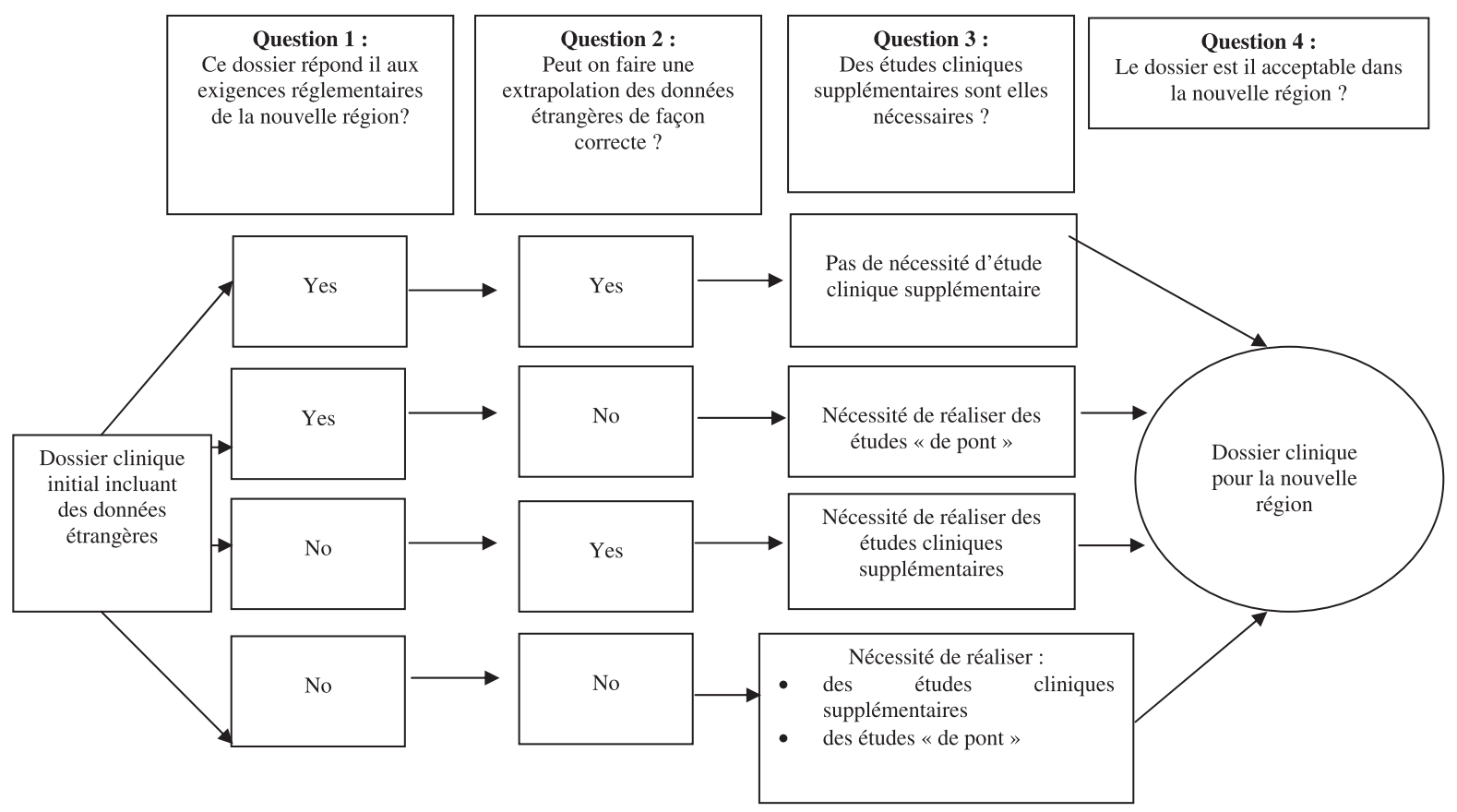

Fig. 1. Méthode d'évaluation d'un dossier clinique contenant des données étrangères (d'après Appendix B - ICH-E5).

hétérogènes dans un contexte tout aussi divers. Les résultats des études cliniques ne sont pas, de ce fait et de façon générale, directement transposables à la pratique. Les sources de variation interethniques de réponse aux médicaments ont fait l'objet de nombreuses revues. ${ }^{[4]}$ Plusieurs éléments liés à l'origine étrangère des études peuvent accentuer les difficultés de transposition. Ces éléments sont répertoriés et discutés par ailleurs. ${ }^{[5]}$ Qu'il s'agisse de différences ethniques, épidémiologiques, culturelles et sociales entre populations cibles et populations étudiées ou de particularités dans l'organisation des systèmes de santé et d'accès au soin entre pays voire de différences de prise en charge thérapeutique, elles peuvent entraîner des écarts d'effet des médicaments pouvant aller jusqu'à inverser, pour une population donnée, un rapport bénéfice/risque estimé dans une autre. Ainsi en est-il de l'aspirine dont le rapport bénéfice/risque se révèle défavorable dans l'indication de prévention des accidents vasculaires cérébraux au Japon alors qu'il est notoirement considéré comme favorable dans les pays occidentaux. ${ }^{[6]}$ La question de la transposabilité des études étrangères est donc un problème méthodologique actuel qui, si l'on souhaite réduire le degré d'incertitude pour prédire l'effet thérapeutique d'un médicament, doit être évoquée et analysée de façon explicite à différentes étapes.

A quel moment la question mérite-t-elle d'être posée? Par qui ? Sur quels éléments peut-on en juger? Dans quel pourcentage de dossiers la question est-elle soulevée? Quelles pourraient être les conséquences d'une erreur d'appréciation? Quelles sont les conséquences d'une transposabilité non assurée sur les déci- sions médico-administratives? Comment améliorer la situation? Tels sont les éléments que les participants à cette table ronde ont choisi d'aborder en limitant l'examen de ces questions aux deux moments clés du parcours du médicament en France : lors de l'AMM (Autorisation de Mise sur le Marché) puis lors du passage en Commission de la Transparence.

\section{Utilisation des études étrangères et nécessaire besoin de transposition de leurs résultats au moment de l'AMM}

Pour juger de l'acceptabilité des résultats d'études étrangères en vue de l'obtention de l'AMM, il convient de déterminer si des différences entre les résultats des études étrangères et ceux attendus dans la pratique clinique dans les pays pour lesquels l'AMM est demandée sont de nature à compromettre le caractère globalement favorable du rapport bénéfice/risque dont l'évaluation est l'objectif principal de la Commission Européenne d'Evaluation des Produits de Santé.

C'est dans cette perspective qu'une recommandation a été élaborée par la conférence internationale d'harmonisation (ICHE5) dont les objectifs sont décrits dans le tableau I.

Cette recommandation décrit également les étapes qui permettent à l'autorité réglementaire d'évaluer l'acceptabilité de données étrangères dans le cadre d'une autorisation de mise sur le marché. Ces étapes sont au nombre de 4 et un schéma fourni en annexe de la recommandation les résument (figure 1). 
Tableau I. Objectifs de la Recommandation ICH-E5.

- Décrire les caractéristiques des données cliniques étrangères qui faciliteront leur extrapolation à des populations différentes et renforceront leur acceptabilité pour leur enregistrement en tant que nouveau médicament dans une région différente.

- Décrire les stratégies réglementaires qui permettront de minimiser la duplication de données cliniques et faciliteront leur acceptabilité dans une nouvelle région.

- Décrire l'utilisation de «bridging studies » (études de pont), dont la réalisation permettra l'extrapolation de données cliniques étrangères à une nouvelle région.

- Décrire des stratégies de développement susceptibles de caractériser les facteurs « ethniques » qui peuvent affecter l'efficacité, la sécurité, la posologie et les modalités de dosage d'un médicament.

L'évaluation initiale quant à la recevabilité du dossier d'un point de vue strictement réglementaire peut donc aboutir à des exigences d'essais cliniques supplémentaires, en particulier : i) dans des sous-populations spécifiques, telles que les insuffisants rénaux ou hépatiques, ii) avec des comparateurs adéquats pour la nouvelle région où l'approbation est sollicitée, iii) ou encore des études d'interactions.

La deuxième étape de cette évaluation consiste à évaluer la sensibilité du médicament à des facteurs dit «ethniques» susceptibles de modifier la nature du rapport risque-bénéfice dans des populations différentes de celles initialement inclues. La recommandation distingue dans cette optique : i) des facteurs intrinsèques qui caractérisent la population cible (âge, sexe, poids, taille, co-morbidités, polymorphisme génétique du métabolisme des patients visés), ii) des facteurs extrinsèques qui concernent l'environnement de la prescription (définition et signification de la pathologie visée dans le contexte culturel régional, facteurs socioéconomiques, différences de pratique médicale ou de stratégie thérapeutique etc.).

Ainsi, ces deux étapes sont déterminantes pour savoir dans quels cas des études «de pont» («bridging studies ») sont nécessaires pour obtenir l'AMM dans une nouvelle région à partir de données initialement recueillies dans une autre région. Selon les cas, ces études peuvent être des études pharmacologiques, de pharmacocinétique ou de pharmacodynamie (PK/PD) ou des études cliniques.

Un exemple d'une telle situation déjà publiế[ ${ }^{[7]} \mathrm{a}$ été rapporté par l'un des membres de la table ronde avec le développement du zolmitriptan enregistré dans un premier temps en Europe puis au Japon. La figure 2 montre comment une extrapolation des données de phase III recueillies dans les pays européens a été acceptée après la réalisation d'une étude « de pont» de pharmacocinétique.

Les conséquences de l'introduction des recommandations ICH-E5 ont été analysées et commentées, en particulier au Japon. ${ }^{[8,9]}$

En Europe, malgré cette méthodologie claire et précise, il est à noter que, jusqu'à présent, la question de la transposabilité pour des facteurs «ethniques » n'a pas représenté à l'EMEA (European

\begin{tabular}{|c|c|c|}
\hline & JAPON & EUROPE \\
\hline Phase I & $\begin{array}{l}\text { Etude d'administration } \\
\text { simple et répétée }\end{array}$ & $\begin{array}{l}\text { Etude d'administration } \\
\text { simple et répétée }\end{array}$ \\
\hline Phase II & 1 étude dose-réponse & 2 études dose-réponse \\
\hline Phase III & Approbation 4 & $\begin{array}{l}1 \text { étude versus placebo } \\
1 \text { étude long-terme }\end{array}$ \\
\hline
\end{tabular}

Fig. 2. Enregistrement du zolmitriptan au Japon.

Agency for the Evaluation of Medicinal Products), un facteur limitant dans la délivrance d'AMM et qu' aucune étude de pont n'a été demandée par l'autorité d'enregistrement depuis l'avènement des recommandations.

\section{Utilisation des études étrangères et nécessaire besoin de transposition de leurs résultats au moment du passage en transparence}

A ce stade, la question de la transposabilité se pose globalement devant les mêmes données et avec les mêmes critères que lors du passage en AMM mais dans une autre optique. Le médicament ayant obtenu l'AMM est considérée comme potentiellement bénéfique pour les patients français définis par l'indication. Il s'agit alors de savoir si les différences entre les résultats des études étrangères et ceux attendus en pratique clinique sur le territoire français sont de nature à modifier substantiellement l'intérêt de santé publique (ISP) et la population cible pour laquelle l'admission au remboursement se justifie, le niveau de SMR (Service Médical Rendu) et d'ASMR (Amélioration du Service Médical Rendu) susceptibles d'influencer l'ensemble des éléments des avis de la Transparence, la question de la transposabilité est abordée explicitement, de façon systématique, au cours de l'évaluation de l'ISP qui se décompose en 2 étapes : i) la première étape consiste à prédire l'ISP attendu théorique en projetant les résultats des essais à partir des données épidémiologiques françaises disponibles : quel nombre d'évènements éviterait-on en France si tout 
se passait dans la pratique comme dans les essais, en faisant l'hypothèse que l'ensemble de la population cible (et uniquement elle) serait rejointe? Sous réserve que l'on puisse disposer du modèle d'effet du médicament et de données épidémiologiques fiables, cette première étape devrait être quantitative. L'origine étrangère des études pouvant être source de différences entre population étudiée et population cible; il s'agit donc de déterminer en quoi l'écart de représentativité peut influencer l'effet attendu du médicament sur la population cible française ? ii) la seconde étape cherche à prédire l'ISP attendu en pratique réelle en corrigeant l'ISP attendu théorique en fonction des différences qui séparent les conditions de l'essai et celles de la pratique clinique française. Les éléments analysés sont essentiellement les facteurs extrinsèques de l'ICH-E5 (en tenant compte des spécificités, nationales telles que modalités de prise en charge, accès aux soins et alternatives thérapeutiques disponibles, médicamenteuses ou non). Cette étape se réduit pour l'instant à une démarche heuristique : estce que l'incertitude générée est suffisante pour mettre en doute le service rendu par le médicament? Une perte d'effet est-elle prévisible et suffisante pour diminuer l'intérêt du médicament?

Parmi les nombreux exemples de difficultés de transposition liées au caractère étranger des études qui ont été discutés au cours de la table ronde, nous en rapporterons ici quelques uns, choisis soit en raison de l'importance de leur impact prévisible, soit en raison de l'incertitude qu'ils génèrent.

- Les statines ont été prises en exemple pour illustrer la question posée par les différences entre population étudiée et population cible. Le poids de santé publique des maladies cardiovasculaires étant variable d'un pays à l'autre, ${ }^{[10]}$ le gain à attendre d'une même intervention varie selon les pays et les stratégies de prévention diffèrent en conséquence. L'essai WOSCOPS ${ }^{[11]}$ a particulièrement alimenté les discussions qui, volontairement, ont fait abstraction de résultats des essais de prévention primaire les plus récents : dans quelle mesure peut-on extrapoler l'effet obtenu avec la pravastatine chez des patients écossais de sexe masculin à une population française, de sexe féminin pour moitié, dont le risque cardiovasculaire est réputé bien moindre ? ${ }^{[12]}$ Il pourrait paraître paradoxal de mettre en doute la transposabilité de données portant sur un sujet aussi bien étudié que celui des statines en prévention cardiovasculaire. En effet, ce domaine a été largement exploré et l'efficacité des statines particulièrement documentée grâce à de grandes études conduites dans plusieurs pays, aux résultats concordants et de fort niveau de preuve. Les dyslipidémies sont des facteurs de risque cardiovasculaires reconnus dans des populations très diverses. ${ }^{[13,14]}$ De multiples études épidémiologiques ont montré que le risque relatif associé à une élévation des taux de cholestérol est comparable quelle que soit la population concernée. Plusieurs essais thérapeutiques de longue durée, portant sur des populations hétérogènes en termes de niveau de risque, ont
Tableau II. Effets des statines sur la fréquence de l'infarctus du myocarde mortel ou non.

\begin{tabular}{lccccc}
\hline Etudes & LDL & IdM Pbo & IdM St & RRR & NNT \\
\hline 4S & 4,7 & 49 & 34 & $27 \%$ & 60 \\
CARE & 3,50 & 26 & 20 & $24 \%$ & 166 \\
LIPID & 3,80 & 17 & 12 & $25 \%$ & 200 \\
HPS & 3,40 & 24 & 17 & $27 \%$ & 140 \\
WOSC & 4,80 & 16 & 11 & $31 \%$ & 200 \\
ASCO & 3,44 & 9,4 & 6,0 & $36 \%$ & 300 \\
CARD & 3,10 & 14,7 & 9,4 & $36 \%$ & 200 \\
AFCA & 3,90 & 5,6 & 3,3 & $40 \%$ & 500 \\
\hline
\end{tabular}

LDL : low density lipoprotein, IdM : infarctus du myocarde, Pbo : groupe placebo, St : groupe statine, RRR : réduction du risque relatif, NNT : nombre nécessaire de patients à traiter.

permis de conclure à des réductions similaires du risque relatif de morbi-mortalité (tableau II). Des fonctions de risque ont été établies et validées qui permettent de prédire le bénéfice d'une modification des paramètres lipidiques associée à un traitement (PROCAM, FRAMINGHAM, SCORE). Ces fonctions de risque devraient permettre d'estimer l'impact de santé publique soit de façon prévisionnelle, sur la population cible théorique, soit sur la population rejointe, en situation de post-inscription. Chercher à prédire les effets thérapeutiques de la pravastatine en prévention primaire dans la population française à partir de l'étude WOSCOPS c'est d'abord estimer le bénéfice absolu à en attendre en tenant compte du risque de base de cette population ( $1^{\text {re }}$ étape); c'est ensuite tenir compte des différences entre les conditions de l'étude et la pratique médicale française ( $2^{\mathrm{e}}$ étape). Le risque de base de la population française laisse espérer un bénéfice absolu dans notre pays relativement faible car les patients à haut risque ressemblant aux hommes écossais de l'étude WOSCOPS sont relativement peu nombreux et le traitement des patients à bas risque, qui y sont majoritaires n'a que peu d'intérêt pour la collectivité. L'impact attendu théorique est donc faible. De plus, les données concernant les conditions d'utilisation des statines en France (caractéristiques des patients traités et durée des traitements), ${ }^{[15]}$ sans remettre en cause leur efficacité potentielle, ne font qu' accroître le doute sur leur impact en pratique réelle.

- L'exemple du Traitement Hormonal de la Ménopause (THM) a été évoqué pour illustrer les difficultés rencontrées lorsque les médicaments évalués ne correspondent pas aux traitements utilisés en situation naturelle. En 2003-2004, après avoir accepté de transposer pendant de nombreuses années les bénéfices du THM à partir de données anglo-saxonnes portant sur des médicaments très peu utilisés en France (estrogènes équins + acétate de médroxyprogestérone), les autorités françaises ont été conduites à transposer, sur la base essentielle de deux études anglo-saxonnes ${ }^{[16,17]}$ les risques liés à l'utilisation de ces mêmes THM (augmentation du risque de survenue de cancers du 
sein, d'accidents coronariens, d'accidents vasculaires cérébraux et d'accidents thrombo-emboliques veineux) pour élaborer les recommandations actuellement en vigueur. ${ }^{[18-20]}$ Bien qu'il existe des arguments physiopathologiques pour penser que les formes transdermiques d'estrogènes exposent moins au risque cardiovasculaire que les estrogènes équins per os (potentiellement moindre risque de maladie thromboembolique veineuse, voire d'infarctus du myocarde et d'accidents vasculaires cérébraux) et des arguments épidémiologiques pour penser qu'en association avec le $17-$ $ß$ estradiol, la progestérone naturelle exposerait moins au risque de cancer du sein que les progestatifs de synthèse, les THM à la française ne bénéficient pas d'études d'un niveau de preuve comparable aux études étrangères, pour faire modifier les choix et les stratégies thérapeutiques découlant des données anglo-saxonnes. L'exemple du THM illustre notamment le fait que, des résultats d'études étrangères portant sur le risque seront à ce jour probablement plus facilement admis par les autorités de santé que des résultats sur les bénéfices.

- La fibrinolyse dans les AVC (accidents vasculaires cérébraux) a servi à illustrer les difficultés à transposer des résultats d'études étrangères lorsqu'il existe des doutes sur leur reproductibilité dans le système de soins français. Les AVC sont, dans un bon nombre de pays, un enjeu de santé publique majeur, à l'origine d'un grand nombre de décès et de handicaps. Un essai clinique américain positif portant sur le r-tPA, utilisé en IV (intra veineuse) dans les 3 premières heures suivant un accident ischémique, a été publié en 1996. ${ }^{[21]}$ Des essais ultérieurs, réalisés, notamment en Europe, ont été non conclusifs ${ }^{[22]}$ voire démonstratifs d'un sur-risque hémorragique (ECASS II). En 1997, 7 des revues systématiques confirmaient l'intérêt du traitement fibrinolytique. ${ }^{[23]}$ Il existe depuis un consensus pour penser que la fibrinolyse peut être un traitement intéressant si elle est utilisée dans les conditions optimales mais aussi très dangereux dès que l'on s'écarte de ces conditions. Prédire les effets thérapeutiques du r-tPA en population française à partir de la méta-analyse réclame donc une attention particulière à la transposabilité des conditions d'utilisation. Il s'agit alors moins de s'interroger sur la reproductibilité de l'essai NINDS sur les malades français dès lors que les conditions optimales seraient réunies que de chercher à savoir quel serait l'impact d'une diffusion du r-TPA en France compte tenu des possibilités et des pratiques de prise en charge actuelles des AVC ? Un registre réalisé au Canada dans la vie réelle chez des patients non sélectionnés (CASES) a permis de confirmer des résultats similaires à ceux de l'essai NINDS avec un taux de saignement faible. ${ }^{[24]}$ En France, il existe plusieurs facteurs limitant les possibilités de transposition : insuffisance des capacités de triage, des moyens d'acheminement, rareté des structures neurovasculaires, des moyens d'imagerie, éducation insuffisante des patients, de l'entourage, des médecins... Malgré les efforts récents réalisés en France pour développer les structures neurovasculaires d'urgence et codifier la prise en charge des AVC, l'impact prévisible de cette technique reste donc incertain dans l'état actuel. Dans le meilleur des cas, en admettant que la fibrinolyse soit utilisée dans les conditions idéales décrites dans les recommandations, son impact favorable ne pourrait être que faible compte tenu de la très faible proportion de patients susceptibles d'accéder au traitement en temps et en heure (moins de $10 \%$ des patients éligibles). Dans une hypothèse moins favorable où les conditions d'utilisation ne seraient pas optimales, on ne pourrait pas exclure un impact de santé publique négatif. L'exemple du r-TPA montre d'une part que la prédiction des effets thérapeutiques d'un médicament en population française est quelquefois indissociable de l'action de santé publique à mener concomitamment à sa mise à disposition, action qui consiste ici à adapter le système de santé afin que le médicament puisse exercer au mieux son effet. Il montre d'autre part que, l'incertitude générée par la mise à disposition de médicament de ce type, à risque sérieux, à index thérapeutique étroit et dont l'effet est fortement dépendant des conditions d'application, doit faire l'objet d'une mesure d'impact dans des conditions observationnelles dans le pays transpositeur.

- Pour illustrer le fait que les critères de jugement des essais sont à l'origine de difficultés de transposition, l'essai pivot portant sur le palivizumab a été discuté. ${ }^{[25]}$ La démonstration de l'efficacité de ce médicament pour prévenir les infections à virus respiratoire syncitial (VRS) reposait sur une étude étrangère utilisant comme critère principal l'incidence des hospitalisations liées au VRS. Les résultats de cette étude étaient d'autant plus difficiles à transposer à la France que les causes d'hospitalisation pour VRS sont autant médicales que sociales et donc variables selon les pays et qu'elle n'apportait pas de preuves de l'efficacité de ce médicament sur des critères cliniques. Cet exemple est à rapprocher d'autres rencontrés notamment dans certains essais de cardiologie où l'on ne sait pas comment utiliser des résultats portant sur des critères combinés incluant l'hospitalisation alors que les déterminants d'une hospitalisation pour une pathologie donnée varient selon les systèmes de santé.

- Les médicaments anti-infectieux ont été pris comme exemples de difficultés de transposition interrégionale en raison, notamment de différences de résistances bactériennes de même que du fait de l'utilisation d'antibiotiques différents selon les pays.

- Les vaccins, médicaments de santé publique par excellence, ont été cités en tant que classe pour laquelle l'analyse du contexte géographique et la prévision de l'effet populationnel grâce à des modèles de simulation sont indispensables. Dépendant de l'écologie microbienne, un vaccin utile dans un pays peut se trouver inutile dans un autre. La prévalence de la maladie à prévenir et son poids de santé publique, les caractéristiques démographiques et notamment l'importance d'une sous-population particulièrement 
fragile à protéger, la stratégie vaccinale utilisant d'autres vaccins conditionnent l'impact prévisible d'un vaccin dans un pays donné et les décisions qui peuvent être prises à son égard. Ainsi peut-on prévoir un impact plus important d'une vaccination contre le rotavirus au Mexique, où la pathologie est endémique et représente un poids de santé publique conséquent qu'en France où la morbimortalité attribuable à ce virus est faible. L'acceptabilité d'un faible risque lié au vaccin, peut aussi s'en trouver modifié. A titre d'exemple, il a été montré que l'intérêt du vaccin antipneumococcique était différent selon le risque de base des pays considérés, son bénéfice ayant été démontré dans des pays non-industrialisés mais pas dans les pays industrialisés. ${ }^{[26]}$ Toutefois, les différences d'effet des vaccins selon les pays ne sont pas toujours expliquées. Ainsi, l'origine de la différence d' effet du vaccin antiHaemophilus efficace en Finlande et non efficace en Alaska, reste discutée. ${ }^{[27]}$ En France, la prédiction des effets des vaccins dans la population est réalisée systématiquement à l'aide de modèles de simulation par le Comité Technique des Vaccinations (groupe de travail permanent de la section des maladies transmissibles du Conseil supérieur d'hygiène publique de France [CSHPF]) en vue d'établir des recommandations vaccinales, constituant ainsi une étape d'évaluation supplémentaire par rapport aux autres médicaments, avant le passage en Transparence.

Les exemples abordés au cours de la table ronde sont trop nombreux pour être tous développés dans cet article. Parmi ceuxci, il est impossible de ne pas évoquer la question des comparateurs. Encore trop souvent, on ne dispose, pour évaluer les médicaments, que d'études étrangères sans comparateur actif alors qu'il existe des traitements standards qui mériteraient d'être comparés. L'une des difficultés provient du fait que les traitements recommandés et/ou disponibles ne sont pas les mêmes dans tous les pays, parfois pour des raisons médicales. C'est notamment le cas d'antibiotiques qui peuvent être préférables dans certains pays du fait de résistances bactériennes. De plus, les protocoles de comparaison ne sont pas toujours les mêmes que ceux utilisés en France, créant ainsi une incertitude supplémentaire sur la quantité d'effet à attendre d'un médicament : comment transposer des résultats d'une chimiothérapie comportant du 5 FU utilisé selon des modalités différentes de celles des standards européens. ${ }^{[28]}$

D'autres difficultés peuvent provenir de différences culturelles entre pays :[29] différences de définition des états pathologiques ou de valeur pathologique attribuée à ces états et donc de propension à traiter (douleur, Déficit de l'attention avec Hyperactivité [TADA]... ) ; comment juger de l'intérêt d'un médicament actif sur le syndrome métabolique en l'absence d'une définition consensuelle de ce syndrome et compte tenu des différences épidémiologiques et de valeur pathologique associée aux

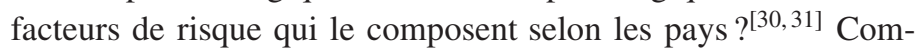
ment transposer des résultats d'études portant sur le reflux gastro- oesophagien, ou sur le sepsis qui n'ont pas les mêmes définitions dans tous les pays ? $^{[32,33]}$ Comment utiliser les résultats d'études nord-américaines sur la pratique de substitution alors que les modalités de l'usage des drogues et la vie quotidienne des toxicomanes sont différentes? Comment, de façon générale interpréter des résultats d'études en termes de qualité de vie, alors que ce concept est variable d'un pays à l' autre, et ce, même si l'utilisation d'instruments de mesures internationaux devrait faciliter les comparaisons interculturelles. ${ }^{[34]} \mathrm{Au}$ travers d'exemples, nous avons voulu illustrer à quel point, pour des raisons diverses, l'origine étrangère des études pouvaient retentir sur l'effet prévisible d'un médicament en population française. La non transposabilité des données peut être seule responsable d'une incertitude à propos de l'effet que peut exercer un médicament sur tout ou partie de la population cible ou d'une présomption forte de perte d'effet ou encore intervenir comme un facteur additif à d'autres limites méthodologiques (plan expérimental biaisé, niveau de preuve insuffisant...). De part les décisions qui sont prises, les conséquences d'une non-transposabilité sont alors notables, autant en termes de santé publique, en conditionnant l'accès au médicament, qu'en termes financier.

Une transposition non assurée dans tout ou partie de la population cible peut conduire la Commission de la Transparence non seulement à modifier sa cotation du SMR et de l'ASMR mais aussi à réduire la population cible admise au remboursement par rapport à celle définie par le libellé de l'AMM et/ou à demander une étude post-inscription dite d'intérêt de santé publique

Sur 108 dossiers examinés entre juin 2004 et juillet 2005 par la Commission de la Transparence, la transposabilité a été jugée suffisamment discutable pour 24 d'entre eux (22\%) par le groupe ISP pour retentir sur les avis. Les difficultés de transposition étaient liées en partie à la présence de données étrangères dans 12 dossiers sur 24 .

Une meilleure documentation de la transposabilité des résultats des études étrangères devrait permettre de réduire le niveau d'incertitude et d'influer ainsi sur les avis scientifiques et les décisions des autorités de santé au moment de l'admission au remboursement.

\section{Pistes de réflexion concernant les actions à mener pour les différents acteurs afin de répondre à la question de la transposabilité des données}

La perspective choisie pour proposer des pistes, a été celle de la chronologie des étapes qui jalonnent le développement d'un médicament :

- phases de développement de nouvelles molécules, 
- constitutions, discussions et évaluations des dossiers d'AMM et de Transparence,

- lors des prescriptions réalisées au lit du malade.

Les actions qui peuvent donc être proposées pour répondre à cette question sont alors :

- Au cours du développement de nouvelles molécules.

Lorsque les entreprises du médicament décident des plans développement, elles devraient : i) autant que possible et de façon générale, inclure des populations suffisamment hétérogènes et larges dans les essais, comprenant, lorsqu'elles souhaitent demander le remboursement en France et à chaque fois qu'il est nécessaire, un contingent de patients français; ii) lorsque cette condition n'est pas remplie, les entreprises devraient se poser systématiquement la question de la transposition et, après consultation des autorités compétentes, inclure le plus tôt possible dans leurs plans de développement les études complémentaires nécessaires; même dans les cas où les études sont présumées de bonne validité interne et théoriquement transposables dans un pays donné (dans les limites des conditions dans lesquelles ont été réalisés les essais), une réflexion devrait être menée systématiquement pour savoir si, des données observationnelles complémentaires, par exemple en utilisant des bases de données médicalisées, seront ou non nécessaires pour répondre à la question de l'admission au remboursement; iii) lorsque des études complémentaires ne paraissent pas nécessaires, les entreprises devraient documenter les éléments permettant de répondre à la question de la transposition de résultats d'études étrangères dans les dossiers d'enregistrement (réponses apportées aux guidelines ICH-E5 en vue de l'AMM pour les médicaments dits « ethniquement sensibles ») et de Transparence (réponses apportées aux questions de la transposition des résultats d'études étrangères à la population française soulevées dans l'évaluation de l'ISPA [Intérêt de Santé Publique Attendu] ou dans l'évaluation de la place du médicament dans la stratégie thérapeutique choix du comparateur); la fourniture de modèles pour apprécier l'ISPA ou des données des essais permettant cette modélisation est hautement souhaitable.

Quel que soit le cas de figure, les entreprises qui souhaitent voir rembourser en France des médicaments dits « ethniquement sensibles » notamment à partir de résultats d'études étrangères devraient fournir des données descriptives ou réaliser des études épidémiologiques permettant de décrire et de quantifier la population cible française visée par le médicament. Ces données sont nécessaires pour réaliser l'exercice de transposition à la population française.

- Au moment de l'évaluation en vue de l'AMM.
Les guidelines ICH-E5 permettent en théorie de répondre à la question de la transposition de résultats d'études étrangères pour les pays concernés par l'AMM en vue de cet enregistrement. En pratique, il a semblé aux participants à la table ronde que la question paraissait insuffisamment évoquée et surtout insuffisamment explicitée lors du passage en commission d'AMM. Il a été proposé que lorsque les dossiers des médicaments reposent sur des études étrangères, des rapports d'évaluation renseignent systématiquement le lecteur à propos du caractère «ethniquement» sensible ou non du médicament considéré et, qu'en cas de médicament « ethniquement» sensible, un argumentaire soit fourni à propos des réponses apportées aux questions soulevées par les guidelines ICH-E5.

- Au moment de l'évaluation par la Commission de la Transparence en vue de l'admission au remboursement.

Ainsi que cela a été décrit plus haut, les conditions d'admission au remboursement d'un médicament sur le territoire français nécessitent de répondre à la question de la transposabilité des résultats des études contenues dans le dossier à la population française, notamment du fait du caractère étranger de ces études. Cette question fait partie des méthodes de l'évaluation de l'ISPA.

Afin de permettre aux entreprises de répondre aux règles de cette évaluation et d'estimer le plus précisément possible l'impact attendu du médicament en pratique réelle sur le territoire français à partir de résultats d'études étrangères, plusieurs pistes devraient être envisagées :

i) la Commission de la Transparence et son groupe de travail ISP devraient communiquer aux entreprises du médicament ses méthodes d'évaluation de l'ISPA et élaborer des recommandations pratiques pour transposer les résultats des essais cliniques à la vie réelle en envisageant la question des études étrangères :

- les méthodes de modélisation et leurs critères de qualité, le type d'hypothèses à formuler devraient notamment être décrits :

- les situations qui devraient faire envisager des études postinscription en raison d'une transposabilité non assurée devraient être communiquées;

ii) d'autre part, la transposition des résultats des études étrangères à la population cible française nécessite de bien connaître cette population; à plusieurs reprises, l'insuffisance de données épidémiologiques françaises a été notée ; plusieurs pistes ont été soulevées pour améliorer cette situation :

○ une première piste consisterait à centraliser et à rendre accessibles les données épidémiologiques existantes;

- la seconde piste consisterait à améliorer la production de connaissances en épidémiologie descriptive : 1) des incitations devraient être données pour que les chercheurs 
académiques (épidémiologistes de l'INSERM, hospitalouniversitaires... ) réalisent ces enquêtes de façon régulière afin que les données épidémiologiques françaises sur les maladies puissent être disponibles et à jour, permettant d'estimer le fardeau des maladies visées en DALYs (Disability Adjusted Life Years); 2) des priorités de thèmes devraient être établies par l'autorité de santé ; 3) des enquêtes spécifiques sur les populations cibles des médicaments devraient par ailleurs être réalisées par les entreprises ( $c f$. supra).

- Au moment de la prescription « au lit du malade».

Pour prendre des décisions thérapeutiques, le prescripteur dispose notamment de référentiels sur les médicaments (RCP [résumé des caractéristiques du produit], avis de la Transparence) et de recommandations pour la pratique clinique. Ces référentiels permettent de guider ses choix dans une situation clinique donnée. Dans la réalité, force est de constater que certaines prescriptions ont lieu hors du cadre des référentiels et hors de l'AMM.

Les résultats des études étrangères réalisées sur le médicament sont généralement encore moins automatiquement transposables à un patient donné qu'à une population et à des conditions de prise en charge plus ou moins différentes de celles des études.

Plusieurs pistes peuvent être proposées pour améliorer la prise en compte de la transposabilité d'études étrangères dans la décision de prescription (dans et hors de l'AMM) :

- en cas de transposabilité non assurée, susceptible de modifier de façon substantielle les performances d'un médicament, une mention pourrait être notée dans le RCP et/ou dans l'avis de la Transparence;

- la transposabilité des données d'études étrangères pourrait être explicitée dans les recommandations pour la pratique clinique (RPC) ; les RPC devraient davantage tenir compte de la pratique réelle en France et des différences escomptées avec les résultats des études étrangères : elles devraient ainsi mieux documenter la validité externe des études qui est généralement inversement proportionnelle à leur validité interne et distinguer les recommandations de fort niveau de preuve avec une forte validité interne mais une faible validité externe;

- le prescripteur qui choisit de prescrire hors AMM en se fondant sur les données actuelles de la science (articles scientifiques) devrait être formé et informé des difficultés de transposition et prendre en compte systématiquement ce critère avant de prendre sa décision; un tel critère pourrait figurer dans les justificatifs demandés aux prescripteurs hospitaliers de médicaments hors GHS dans le cadre des contrats de bon usage.

\section{Conclusion}

La question de la transposabilité ne cesse de prendre de l'importance dans le cadre du développement de nouveaux médicaments. En effet, jusqu'à il y a peu de temps, tous les efforts se sont concentrés sur la qualité des preuves de la démonstration expérimentale de l'efficacité et de la tolérance. A présent, au-delà de cette démonstration, il paraît nécessaire de prédire de la façon la plus précise possible, les effets attendus des médicaments sur les populations cibles. La mise en œuvre des pistes proposées devrait permettre d'améliorer la précision de l'estimation de l'effet attendu des nouveaux médicaments ou de nouvelles indications. Lorsque ces pistes n'auront pas été mises en place, il sera alors nécessaire de réaliser des études de suivi afin de confirmer en situation réelle de traitement les hypothèses faites d'obtention du Service Médical Attendu.

\section{Participants}

L. Abenhaïm (Hôpital Cochin, Paris), P. Ambrosi (Hôpital Sainte Marguerite, Marseille), M. Bardou (Faculté de Médecine, Dijon), J-P. Boissel (Faculté de Médecine RTH, Lyon), C. Brun (Novartis, Rueil), A. Castaigne (Astra Zeneca, Créteil), O. Chassany (Hôpital Saint Louis, Paris), F. de Bels (HAS, SaintDenis), R. de Sahb-Berkovitch (Roche, Neuilly-sur-Seine), A. ElHasnaoui (Glaxo Smith Kline, Marly-le-Roi), F. Fagagni (Cemka, Bourg-la-Reine), A. Fourrier-Reglat (Université Victor Segalen, Bordeaux), C. Gastaldi-Meninger (Saint-Denis), J.-M. Goehrs (MSD, Paris), F. Gueffier (Hôpital Louis Pradel, Lyon), J.-M. Hotton (Pfizer, Paris), F. Ichou (Sanofi - Aventis, Paris), P. Lechat (Hôpital Pitié-Salpétrière, Paris), P. Maillère (Servier, Courbevoie), F. Meyer (HAS, Saint-Denis), J. Micallef (Hôpital de la Timone, Marseille), M. Molimard (Hôpital Pellegrin, Bordeaux), T. Moreau-Defarges (Janssen Cilag, Issy-les-Moulineaux), A. Perillat (DGS, Paris), M. Pigeon (CNAMTS, Paris), P. Poitrinal, C. Rey-Quino (AFSSAPS, Saint-Denis), P. Ricordeau (CNAMTS, Paris), J. Ropers (AFSSAPS, Saint-Denis)

\section{Références}

1. Courcier-Duplantier S, Bouhours $\mathrm{P}$, Pinton $\mathrm{P}$, et al. Groupe de travail Attractivite de la France du Leem. A survey on the position of France in international clinical research as assessed by pharmaceutical laboratories. Therapie 2004; 59(6): 629-38

2. Mant D. Can randomised trials inform clinical decision about individual patients? Lancet 1999; 353(9154): 743-6

3. Sculpher MJ, Pang FS, Drummond MF, et al. Generalisability in economic evaluation studies in health care: a review and case studies. Health Technology Assessment (Winchester, England). 8(49):iii-iv, 1-192, 2004 Dec. 
4. Bjornsson TD, Wagner JA, Donahue SR, et al. Review and assessment of potential sources of ethnic differences in drug responsiveness. The Journal of Clinical Pharmacology 2003; 43: 943-67

5. Martinez MN, McGilveray I. AAPS/RAPS/CAPRA collaborative program: exploring the challenges of drug regulation in a global environment: clinical concerns. Aaps Pharmsci. 2003; 5(4): E27

6. Morimoto T, Fukui T, Lee TH, et al. Application of US guidelines in other countries: aspirin for the primary prevention of cardiovascular events in Japan. Am J Med 2004; 117: 459-68

7. Sakai F, Iwata M, Tashiro K, et al. Zolmitriptan is effective and well tolerated in Japanese patients with migraine: a dose-response study. Cephalalgia 2002; 22(5): 376-83

8. Shunsuke Ono, Osamu Kato, Kiichiro Tsutani, et al. Utilisation of foreign clinical data in Japanese new drug approval review: on what basis did the regulatory agency accept them? International Journal of Pharmaceutical Medicine 2004; 18(3): 159-65

9. Liu JP, Chow SC. Bridging studies in clinical development. J Biopharm Stat 2002; 12(3): 359-67

10. Yusuf S, Reddy S, Ounpuu S, et al. Global Burden of cardiovascular diseases Part II: variations in cardiovascular disease by specific ethnic groups and geographic regions and prevention strategies. Circulation. 2001; 104(23): 2855-64

11. West of Scotland Coronary prevention Study: implications for clinical practice The WOSCOPS Study Group. Eur Heart J 1996; 17(2): 163-4

12. Tunstall-Pedoe H, Kuulasmaa K, Amouyel $\mathrm{P}$, et al. Myocardial infarction and coronary deaths in the World Health Organization MONICA Project. Registration procedures, event rates, and case-fatality rates in 38 populations from 21 countries in four continents. Circulation 1994; 90(1): 583-612

13. Ounpuu S, Negassa A, Yusuf S. INTER-HEART STUDY: A global study of risk factors for acute myocardial infarction. Am Heart J 2001; 141(5): 711 21

14. Ezzati M. How can cross-country research on health risks strengthen interventions? Lessons from INTERHEART. Lancet 2004; 364(9438): 912-4

15. Instauration des traitements médicamenteux hypolipémiants en France en 2002, Rev Med Ass Maladie 2003; 34,4 :221-231

16. Rossouw JE, Anderson GL, Prentice RL, et al. Writing Group for the Women's Health Initiative Investigators. Risks and benefits of estrogen plus progestin in healthy postmenopausal women: principal results from the Women's Health Initiative randomized controlled trial. JAMA 2002; 288(3): 321-33

17. Beral V. Breast cancer and hormone-replacement therapy in the Million Women Study. Lancet 2003; 362(9382): 419-27

18. Mise au point sur le traitement hormonal substitutif de la ménopause (THS). Afssaps Janvier 2003 [http: //www . afpssaps . sante. fr]

19. Mise au point actualisée sur le traitement hormonal substitutif de la ménopause (THS). Afssaps. Décembre 2003 [http: //www . afpssaps . sante . fr]

20. Rapport d'orientation sur les traitements hormonaux substitutifs de la ménopause. Anaes/Afssaps. 11 mai 2004 [http:// www.afpssaps.sante.fr]
21. Sila CA, Furlan AJ. Therapy for acute ischemic stroke: the door opens. Interpreting the NINDS rt-PA stroke study. Cleve Clin J Med 1996; 63(2): $77-9$

22. Hacke W, Donnan G, Fieschi C, et al. ATLANTIS Trials Investigators. ECASS Trials Investigators. NINDS rt-PA study group investigators. Association of outcome with early stroke treatment: pooled analysis of ATLANTIS, ECASS, and NINDS rt-PA stroke trials. Lancet 2004; 363(9411): 768-74

23. Wardlaw JM, Warlow CP, Counsell C. Systematic review of evidence on thrombolytic therapy for acute ischaemic stroke. Lancet 1997; 350: 607-14

24. Demchuk AM, Hill MD, Barber PA, et al. Importance of early ischemic computed tomography changes using ASPECTS in NINDS rtPA stroke study. Stroke 2005; 36(10): 2110-5

25. The IMPact-RSV study group. Palivizumab, humanized respiratory syncytial virus monoclonal antibody, reduces hospitalization from respiratory syncytial virus infection in high risk infants. Pediatrics 1998; 102(3): 531-7

26. Watson L, Wilson BJ, Waugh N. Pneumococcal polysaccharide vaccine: a systematic review of clinical effectiveness in adults. Vaccine 2002; 20(17-18): 2166-73

27. Shapiro ED. Protective efficacy trials. In: Ellis RW. Development and clinical uses of haemophilus b conjugate vaccines. New York, NY: Marcel Dekker; 1994 may; 339-56

28. Efficacy of intravenous continuous infusion of fluorouracil compared with bolus administration in advanced colorectal cancer. Meta-analysis Group In Cancer. J Clin Oncol 1998 Jan;16(1): 301-8

29. Balant LP, Balant-Gorgia EA. Cultural differences: implications on drug therapy and global drug development. Int J Clin Pharmacol Ther 2000; 38(2): 47-52

30. American Heart Association, National Heart, Lung and Blood Institute, Grundy SM, et al. Diagnosis and management of metabolic syndrome. Cardiol rev 2005; 13(6): 322-7

31. Patel A, Huang KC, Janus ED, et al. Is a single definition of the metabolic syndrome appropriate? A comparative study of the USA and Asia. Atherosclerosis 2006; 184(1): 225-32

32. Veldhuyzen Van Zanten SJ, Flook N, Chiba N, et al. An evidence-based approach to the management of uninvestigated dyspepsia in the era of Helicobacter pylori. Canadian Dyspepsia Working Group. CMAJ 2000; 162 (12 suppl): S3-23

33. Graf J, Doig GS, Cook DJ, et al. Randomized, controlled clinical trials in sepsis: has methodological quality improved over time? Crit Care Med 2002; 30(2): 461-72

34. Bullinger M, Anderson D, Cella D, et al. Developping and evaluating crosscultural instruments for minimal requirements to optimal models. Qual Life Res 1993; 2: 451-9

Correspondance et offprints : Myriam Zylberman, Lilly France, 13 rue Pagès, 92158 Suresnes Cedex, France.

E-mail : zylberman_myriam@lilly.com 